\title{
Ear Drops, Solution in Single-dose Container
}

National Cancer Institute

\section{Source}

National Cancer Institute. Ear Drops, Solution in Single-dose Container. NCI Thesaurus.

Code C150066.

Medicinal product consisting of an ear drops solution presented in a single-dose container. 